\title{
Original
}

\section{Top 100. Revisión de los artículos más citados sobre cirugía laparoscópica del páncreas}

\author{
Alba Manuel-Vázquez ${ }^{a, *}$, José Ramón Oliver-Guillén ${ }^{b}$, Raquel Latorre-Fragua ${ }^{a}$, \\ Ana Palomares Cano ${ }^{c}$, Mario Serradilla Martín ${ }^{c}$ y José Manuel Ramia ${ }^{a}$ \\ ${ }^{a}$ Cirugía General y Digestiva, Hospital Universitario de Guadalajara, Guadalajara, España

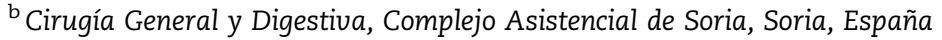 \\ 'Cirugía General y Digestiva, Hospital Universitario Miguel Servet, Zaragoza, España
}

\section{INFORMACIÓN DEL ARTÍCULO}

Historia del artículo:

Recibido el 19 de abril de 2020

Aceptado el 2 de mayo de 2020

On-line el $\mathrm{xxx}$

Palabras clave:

Bibliometría

Artículo

Cita

Cirugía

Páncreas

Laparoscopia

\begin{abstract}
R E S U M E N
Introducción: El número de citas se considera un indicador indirecto del mérito de un artículo, revista o investigador, aunque no es un método infalible para determinar la calidad científica. Nuestro objetivo es determinar las características de los artículos más citados sobre páncreas y laparoscopia.

Métodos: Realizamos una búsqueda de todos los artículos publicados en cualquier revista sobre páncreas y laparoscopia hasta septiembre de 2019 y seleccionamos los 100 artículos más citados. Registramos el número de citas, la revista, el año de publicación, el cuartil, el factor de impacto, la institución, el país, el tipo de artículo de los autores, el tipo de cirugía, el tema y el área.

Resultados: El top 100 suma 10.970 citas. La revista con más artículos es Surgical Endoscopy y 2007 es el año con el mayor número de artículos en el top 100. El porcentaje de publicaciones de América y Europa es similar. Las series de casos son el tipo de artículo más frecuente, los resultados/morbilidad es el tema más discutido y la pancreatectomía distal es el tipo de cirugía más frecuente.

Conclusiones: Este estudio bibliométrico sobre páncreas y laparoscopia está condicionado por el factor tiempo, ya que la laparoscopia ha llegado más tarde a la cirugía pancreática, probablemente debido a la morbimortalidad asociada a la cirugía pancreática y a la necesidad de una alta especialización en este campo. La literatura es reciente y escasa. Se necesitan más estudios y de mayor calidad en este campo.
\end{abstract}

(C) 2020 AEC. Publicado por Elsevier España, S.L.U. Todos los derechos reservados.

\footnotetext{
* Autor para correspondencia.

Correo electrónico: alba_manuel_vazquez@hotmail.com (A. Manuel-Vázquez). https://doi.org/10.1016/j.ciresp.2020.05.001

0009-739X/C 2020 AEC. Publicado por Elsevier España, S.L.U. Todos los derechos reservados.
} 
Keywords:

Bibliometrics

Article

Citation

Surgery

Pancreas

Laparoscopy

\section{The top 100. Review of the most cited articles on pancreas and laparoscopy}

\section{A B S T R A C T}

Introduction: The number of citations is considered as an indirect indicator of the merit of an article, journal or researcher, although it is not an infallible method to determine scientific quality. Our goal is to determine the characteristics of the articles most cited about pancreas and laparoscopy.

Methods: We performed a search of all articles published in any journal about pancreas and laparoscopy until September 2019 and selected the 100 most cited papers. We recorded number of citations, journal, year of publication, quartil, impact factor, institution, country, authors type of paper, type of surgery, topic and area.

Results: The top 100 citations account 10,970 citations in total. The journal with the most articles is Surgical Endoscopy and 2007 is the year with the highest number of articles in the top 100 citations. The percentage of publications from America and Europe are similar.

Case series is the most frequently paper, outcomes/morbidity is the most frequently discussed topic, and distal pancreatectomy is the most frequently type of surgery.

Conclusions: This bibliometric study on pancreas and laparoscopy is conditioned by the time factor, since laparoscopy has arrived later at pancreatic surgery, probably due to the morbidity and mortality associated with pancreatic surgery and the need for a high specialization in this field. The literature is recent and scarce. More and better-quality studies are needed in this field.

(C) 2020 AEC. Published by Elsevier España, S.L.U. All rights reserved.

\section{Introducción}

El número de citas de un artículo es un método para determinar el impacto de un investigador o de una publicación en la comunidad científica, junto con otros indicadores como el factor de impacto ${ }^{1-8}$. El número de citas se considera un indicador indirecto del mérito de un artículo, revista o investigador ${ }^{1,2,4,5}$, aunque no es un método infalible para determinar la calidad científica.

Los estudios bibliométricos permiten conocer cómo se obtiene la información científica, de dónde procede y cuál es su calidad. Recientemente se han publicado una serie de artículos sobre «citas clásicas» (o los artículos más citados) en diversas especialidades para definir las características que debe tener una publicación para ser admitida en esta lista de selección ${ }^{1-5,7,9}$.

Por otro lado, la bibliografía referente al páncreas y a la laparoscopia es muy escasa, y un estudio bibliométrico sobre la materia nos puede permitir conocer la situación actual sobre el tema e investigar la calidad de los trabajos publicados, así como conocer los campos de conocimiento donde se requiere mayor investigación.

Nuestro objetivo es determinar las características de los artículos más citados sobre páncreas y laparoscopia.

\section{Métodos}

Realizamos una búsqueda de todos los artículos publicados en cualquier revista sobre páncreas y laparoscopia a fecha de 24 de septiembre de 2019, utilizando la aplicación de Thomson
Reuters ISI Web of Science (WEB). Nuestra búsqueda incluyó los siguientes términos: ((Pancreas)) AND ((Surgery)) AND ((Laparoscopy) OR (Video-Assisted Surgery) OR (Natural Orifice Endoscopic Surgery) OR (Robotic Surgical Procedures) OR (Hand-Assisted Laparoscopy)).

La aplicación de Thomson Reuters ISI Web of Science incluye las siguientes bases de datos:

- Web of Science (1990-presente)

- Índice de citaciones de la BIOSIS (2006-presente)

- Avances de BIOSIS (1926-presente)

- Current Contents Connect (1998-presente)

- Índice de innovaciones de Derwent (1980-2009).

- KCI - Base de datos de revistas coreanas (1980-presente)

- MEDLINE $®$ (1950-presente)

- Índice de citas de la ciencia rusa (2005-presente)

- Índice de citas de SciELO (2002-presente)

Seleccionamos los 100 artículos más citados utilizando la categoría «veces citadas» en todas las bases de datos (top 100 citas).

En cada artículo evaluamos: título, número de citas, revista, año de publicación, cuartil (Q), factor de impacto (FI) por año, institución del primer autor, país (si es multicéntrico, elegimos el país del primer autor), número de autores, nombre del primer autor, tipo de artículo, tema, tipo de cirugía y área.

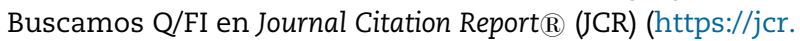
incites.thomsonreuters.com/JCRJournalHomeAction.action) y los seleccionamos según el año. Si no había Q/FI online del año, seleccionamos el primer Q/FI online publicado en JCR (entre paréntesis). 
Seleccionamos la institución del primer autor y el país de acuerdo con el centro/país de trabajo en el momento de la publicación.

Clasificamos el tipo de trabajo como originales, estudios controlados aleatorizados (ECA), revisión, revisión sistemática, metaanálisis, series de casos, estudio de cohortes y un grupo denominado "otros», que incluye casos clínicos, cartas o descripción de técnicas quirúrgicas.

Dividimos el tema del artículo en estadificación, morbilidad, técnica quirúrgica, cirugía laparoscópica vs. cirugía abierta, resultados/morbilidad y cirugía experimental.

El tipo de cirugía se clasificó como duodenopancreatectomía (DP), pancreatectomía total, pancreatectomía distal (PD), cirugía robótica, cirugía pancreática, cirugía paliativa, desbridamiento retroperitoneal videoasistido (VARD) y «sin cirugía", refiriéndose a artículos no relacionados con procedimientos quirúrgicos.

El área de interés se dividió en enfermedad benigna, pancreatitis aguda, pancreatitis crónica, adenocarcinoma, tumor neuroendocrino, neoplasia quística, neoplasia mucosa papilar intraductal (NMPI), neoplasias y lesión benigna/ maligna.

\section{Resultados}

Tras la búsqueda, se analizaron un total de 2.182 artículos. El número total de artículos publicados varía de una revista a otra, ya que algunas revistas han estado en funcionamiento más tiempo que otras.

El top 100 suma 10.970 citas en total (tabla 1). El artículo más citado es de 1994, incluye la primera PD laparoscópica descrita por Gagner y cuenta con 530 citas desde su publicación. Los 3 artículos más citados -Gagner 1994 con 530 citaciones, Mabrut 2005 con 328 y Gagner 1996 con 311- suponen 1.169 citas, más del 10\% del total de este top 100 (1.169/10.970; 10,66\%). Los 27 artículos más citados de este ranking suman 5.527 citas (5.527/10.970; 50,38\% del total).

Según el año, 2007 cuenta con 11 artículos que representan 1.081 citas, mientras que 2010 es el año con mayor cantidad de citas, 1.256 citas y 9 artículos (fig. 1).

La revista con más artículos incluidos en este top 100 es Surgical Endoscopy (30/100; 30\%). En segundo lugar, encontramos Annals of Surgery con 9 y J Am Coll Surg con 8. Cuatro revistas son responsables del $50 \%$ de los artículos incluidos

Tabla 1 - Referencias incluidas en el top 100 de citas sobre páncreas y laparoscopia

\begin{tabular}{|c|c|c|}
\hline Referencia & Primer autor & Número de citaciones \\
\hline Surg Endosc. 1994;8(5):408-10 & Gagner M & 530 \\
\hline Surgery. 2005;137(6):597-605 & Mabrut JY & 328 \\
\hline Surgery. 1996;120(6):1051-4 & Gagner M & 311 \\
\hline Ann Surg. 2012;255(6):1048-59 & Venkat R & 293 \\
\hline Ann Surg. 2008;248(3):438-46 & Kooby DA & 293 \\
\hline Ann Surg. 1995;221(2):156-64 & John TG & 258 \\
\hline Arch Surg. 2010;145(1):19-23 & Kendrick ML & 247 \\
\hline Surg Endosc. 2010;24(7):1646-57 & Giulianotti PC & 246 \\
\hline J Am Coll Surg. 2012;215(6):810-9 & Asbun $\mathrm{HJ}$ & 235 \\
\hline Ann Surg. 1996;223(2):134-40 & Conlon KC & 226 \\
\hline J Am Coll Surg. 2010;210(5):779-85 & Kooby DA & 225 \\
\hline J Am Coll Surg. 2007;205(2):222-30 & Palanivelu C & 193 \\
\hline Ann Surg. 2007;246(1):77-82 & Melotti G & 188 \\
\hline Ann Surg. 1996;223(3):280-5 & Cuschieri A & 163 \\
\hline J Gastrointest Surg. 2007;11(12):1607-21 & Fernández-Cruz L & 161 \\
\hline Ann Surg. 2002;236(2):149-58 & Park AE & 161 \\
\hline Surg Endosc. 2006;20(7):1045-50 & Dulucq JL & 160 \\
\hline J Gastrointest Surg. 2004;8(4):493-501 & Fernández-Cruz L & 145 \\
\hline Surg Endosc. 2008;22(10):2261-8 & Kim SC & 143 \\
\hline World J Surg. 2008;32(5):904-17 & Fernández-Cruz L & 139 \\
\hline Surgery. 2000;128(3):386-91 & Berends & 132 \\
\hline J R Coll Surg Edinb. 1994;39(3):178-84 & Cuschieri A & 129 \\
\hline Surg Endosc. 2011;25(6):2004-9 & Kang CM & 127 \\
\hline J Am Coll Surg. 2010;211(4):503-9 & Jayarama S & 127 \\
\hline J Am Coll Surg. 1997;185(1):33-9 & Callery MP & 124 \\
\hline Br J Surg. 1995;82(8):1127-9 & Fernandez del Castillo C & 124 \\
\hline Surg Endosc. 2011;25(10):3364-72 & Song $\mathrm{KB}$ & 119 \\
\hline Gut. 1978;19(7):672-7 & Cuschieri A & 118 \\
\hline Arch Surg. 2010;145(9):817-25 & Horvath $\mathrm{K}$ & 117 \\
\hline J Gastrointest Surg. 2006;10(1):95-8 & Velanovich V & 117 \\
\hline Langenbecks Arch Surg. 2005;390(2):134-40 & Ayav A & 117 \\
\hline Surgery. 2015;157(1):45-55 & Mehrabi A & 116 \\
\hline Ann Surg. 1998;228(2):182-7 & Minnard EA & 116 \\
\hline J Hepatobiliary Pancreat Surg. 2009;16(6):731-40 & Palanivelu C & 115 \\
\hline Br J Surg. 1992;79(4):317-9 & Shimi S & 115 \\
\hline World J Surg. 2002;26(8):1057-65 & Fernández Cruz L & 113 \\
\hline
\end{tabular}


Tabla 1 (Continuación)

\begin{tabular}{|c|c|c|}
\hline Referencia & Primer autor & Número de citaciones \\
\hline Surg Endosc. 2008;22(5):1334-8 & Eom BW & 112 \\
\hline Surg Endosc. 2004;18(3):407-11 & Edwin B & 107 \\
\hline J Gastrointest Surg. 2011;15(7):1151-7 & Zureikat $\mathrm{AH}$ & 105 \\
\hline Surg Endosc. 2005;19(8):1028-34 & Dulucq JL & 103 \\
\hline HPB (Oxford). 2012;14(11):711-24 & Jin $\mathrm{T}$ & 100 \\
\hline J Laparoendosc Adv Surg Tech A. 2003;13(1):33-6 & Melvin WS & 95 \\
\hline Aust N Z J Surg. $1996 ; 66(6): 414-6$ & Sussman LA & 94 \\
\hline Am J Surg. $2009 ; 198(3): 445-9$ & Cho A & 91 \\
\hline Surg Endosc. 2007;21(4):579-86 & Pierce RA & 90 \\
\hline J Hepatobiliary Pancreat Sci. 2013;20(4):421-8 & Nakamura M & 89 \\
\hline Gut. $1995 ; 36(5): 778-80$ & Rhodes M & 85 \\
\hline Pancreas. 2012;41(7):993-1000 & Pericleous S & 84 \\
\hline J Gastrointest Surg. 2010;14(11):1804-12 & DiNorcia J & 84 \\
\hline Endoscopy. 2007;39(10):881-7. & Ryou M & 84 \\
\hline World J Surg. 2004;28(12):1239-47 & Assalia A & 81 \\
\hline Surg Endosc. 2010;24(7):1533-41 & Kang CM & 80 \\
\hline Surg Endosc. 2004;18(3):402-6 & Shimizu S & 78 \\
\hline Surg Endosc. 2003;17(2):201-6 & Tagaya N & 78 \\
\hline Pancreas. 2010;39(2):160-4 & Narula VK & 77 \\
\hline Surg Endosc. 1994;8(1):57-60 & Soper NJ & 74 \\
\hline J Am Coll Surg. 2015;220(5):831-8 & Dokmak S & 73 \\
\hline Arch Surg. 2004;139(3):270-4 & Jaroszewski DE & 73 \\
\hline Pancreas. 2008;36(2):113-9 & Bucher P & 72 \\
\hline Surg Endosc. 2007;21(3):373-7 & Palanivelu C & 72 \\
\hline Ann Oncol. 2006;17(2):189-99 & Stefanidis D & 71 \\
\hline J Am Coll Surg. 2008;206(3):445-50 & White R & 70 \\
\hline Surg Endosc. 2002;16(9):1358-61 & Fabre JM & 68 \\
\hline J Hepatobiliary Pancreat Surg. 2009;16(1):35-41 & Nakamura Y & 67 \\
\hline Asian J Surg. 2012;35(1):1-8 & Sui CJ & 65 \\
\hline Surg Endosc. 2012;26(2):402-7 & Mehta SS & 64 \\
\hline J Am Coll Surg. 2009;209(6):758-65 & Borja-Cacho D & 64 \\
\hline Cancer J. 2012;18(6):571-6 & Kendrick ML & 62 \\
\hline Surgery. 2001;130(6):1086-91 & Iihara $\mathrm{M}$ & 62 \\
\hline Surg Endosc. 2007;21(12):2326-30 & Pryor A & 61 \\
\hline World J Surg. 2002;26(10):1297-300 & Gramatica L & 61 \\
\hline Surg Endosc. 2000;14(12):1131-5 & Lo $\mathrm{CY}$ & 61 \\
\hline Surg Endosc. 1992;6(3):147-9 & Fletcher DR & 61 \\
\hline Surg Endosc. 2011;25(2):572-6 & Didieu A & 60 \\
\hline Ann Surg Oncol. 2009;16(10):2825-33 & Weber Sm & 60 \\
\hline Surg Today. 2007;37(7):535-45 & Takaori K & 60 \\
\hline Surg Endosc. 2012;26(5):1220-30 & Fox AM & 59 \\
\hline Surgery. 2007;142(3):405-9 & Sa Cunha AS & 59 \\
\hline Semin Laparosc Surg. 1998;5(3):168-79 & Cuschieri A & 59 \\
\hline Br J Surg. 2009;96(2):185-90 & Isla $\mathrm{A}$ & 58 \\
\hline J Gastrointest Surg. 2004;8(8):1068-71 & Shoup M & 58 \\
\hline Surg Endosc. 2011;25(4):1101-6 & Kang CM & 57 \\
\hline Surg Endosc. 2007 Dec;21(12):2262-7 & Palanivelu C & 57 \\
\hline Surg Endosc. 2007 Jan;21(1):103-8 & Sa Cunha AS & 56 \\
\hline World J Gastroenterol. 2012;18(16):1959-67 & Xie $\mathrm{K}$ & 55 \\
\hline J Surg Oncol. 2012;105(4):387-92 & Butturini G & 55 \\
\hline Pancreas. 2009;38(8):867-75 & Navaneethan U & 55 \\
\hline Pancreas. 2011;40(8):1264-70 & Giulianotti PC & 53 \\
\hline Br J Surg. 2010;97(6):902-9 & Rosok BI & 53 \\
\hline Adv Surg. $2009 ; 43: 283-300$ & Merchant NB & 53 \\
\hline Surg Endosc. 2005;19(3):369-73 & Ellsmere J & 53 \\
\hline Surg Laparosc Endosc Percutan Tech. 2008;18(4):340-3 & Matsumoto $\mathrm{T}$ & 52 \\
\hline Am J Surg. 2006;191(4):549-52 & Orsenigo E & 52 \\
\hline Surg Endosc. 2004;18(2):297-302 & Lo CY & 52 \\
\hline Acta Chir Hung. 1997;36(1-4):359-61 & Tihanyi TF & 52 \\
\hline World J Surg. 2006;30(10):1916-9 & Toniato A & 51 \\
\hline Surg Endosc. 2002;16(6):996-1003 & Fernández-Cruz L & 51 \\
\hline Surg Endosc. 1999;13(11):1065-9 & Röthlin MA & 51 \\
\hline Ann Surg. 2008;247(6):938-44 & Rotellar F & 50 \\
\hline Surg Endosc. 1999;13(3):239-45 & Catheline JM & 50 \\
\hline
\end{tabular}



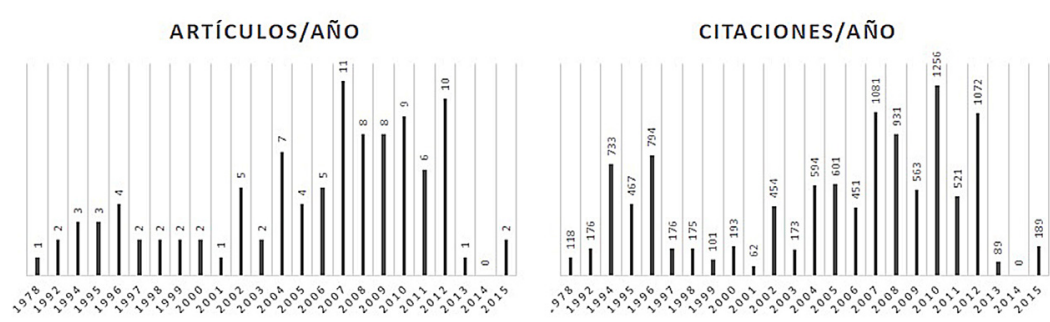

Figura 1 - Número de artículos y citaciones por año en el top 100 de citas sobre páncreas y laparoscopia.

(Surg Endosc 30, Ann Surg 9, J Am Coll Surg 8, Surgery 6, $J$ Gastrointest Surg 6). Las revistas restantes son Pancreas con 5 artículos, World J Surg con 5, Br J Surg con 4, Arch Surg con 3, Gut con 2, J Hepatobiliary Pancreat Surg con 2 y Am J Surg con otros 2 artículos. Hay otras 18 revistas con una sola publicación en este top 100 de citas. El FI de todos los artículos incluidos en este top 100 de citas es 212,7. El 78\% de los artículos se publicaron en revistas Q1.

Según el país, EE. UU. es responsable de 35 publicaciones (35\%), seguido de Francia con 9, Reino Unido y Japón con 8 y España con 6. Si clasificamos los países por continentes, los porcentajes de publicaciones procedentes de América y Europa son similares, 39\% frente a 36\% respectivamente, con mayor dispersión de publicaciones en Europa. Asia es responsable del $22 \%$ de las publicaciones de este top 100 (Japón 8 , China 5, Corea 5, India 4) y Oceanía, del 3\% restante.
Según el centro de trabajo del primer autor, 14 centros suman 40 publicaciones y 4.691 citas. El Memorial SloanKettering Cancer Center es el centro con más referencias incluidas en este top 100 de citas, mientras que Mayo Clinic es el centro con el mayor número de citas. En cuanto al primer autor, solo hay 3 autores con más de un artículo en este ranking. Fernández-Cruz L, cuya labor se desarrolla en el Hospital Clínic de Barcelona, tiene 5 publicaciones con un total de 609 citas ( 5 series de casos), Cuschieri A tiene 4 publicaciones con 469 citas (2 series de casos, una revisión y una retrospectiva quirúrgica) y Palanivelu $C$ tiene 4 artículos con 437 citas (4 series de casos) (tabla 2).

Según el tipo de artículo, 67 publicaciones incluidas en el top 100 de citas son series de casos, lo que supone que este tipo de artículo es el que se cita con más frecuencia (7.422 citas). Siete trabajos son metaanálisis, sumando 802 citas $(5$ publicados en

Tabla 2 - Número de artículos y citaciones según centro y primer autor en el top 100 de citas sobre páncreas y laparoscopia. "Otros" incluye centros con solo un artículo en este top 100

\begin{tabular}{|c|c|c|c|}
\hline Centro & & Artículos & Citaciones \\
\hline Memorial Sloan-Kettering Cancer Center & & 5 & 597 \\
\hline Mayo Clinic & & 4 & 617 \\
\hline Hospital Clínic, Barcelona & & 4 & 448 \\
\hline Gem Hospital & & 4 & 437 \\
\hline Ninwells Hospital and Medical School, University of Dundee & & 4 & 466 \\
\hline Washington University School of Medicine & & 4 & 281 \\
\hline University of Illinois, Chicago & & 2 & 299 \\
\hline Emory University School of Medicine & & 2 & 518 \\
\hline Ohio State University & & 2 & 172 \\
\hline Massachusetts General Hospital & & 2 & 177 \\
\hline Ohio State University & & 2 & 172 \\
\hline Saint Eloi Hospital & & 2 & 132 \\
\hline Ulsan University College of Medicine and Asan Medical Center & & 2 & 262 \\
\hline Queen Mary Hospital, Hong Kong & & 2 & 113 \\
\hline Otros & & 59 & 6.279 \\
\hline Primer autor & Artículos & & Citaciones \\
\hline Fernández-Cruz L & 5 & & 609 \\
\hline Cuschieri A & 4 & & 469 \\
\hline Palanivelu C & 4 & & 437 \\
\hline Kang CM & 3 & & 264 \\
\hline Dulucq JL & 2 & & 263 \\
\hline Gagner M & 2 & & 841 \\
\hline Giulianotti PC & 2 & & 299 \\
\hline Kendrick ML & 2 & & 309 \\
\hline Kooby DA & 2 & & 518 \\
\hline Lo CY & 2 & & 113 \\
\hline Sa Cunha AS & 2 & & 115 \\
\hline
\end{tabular}




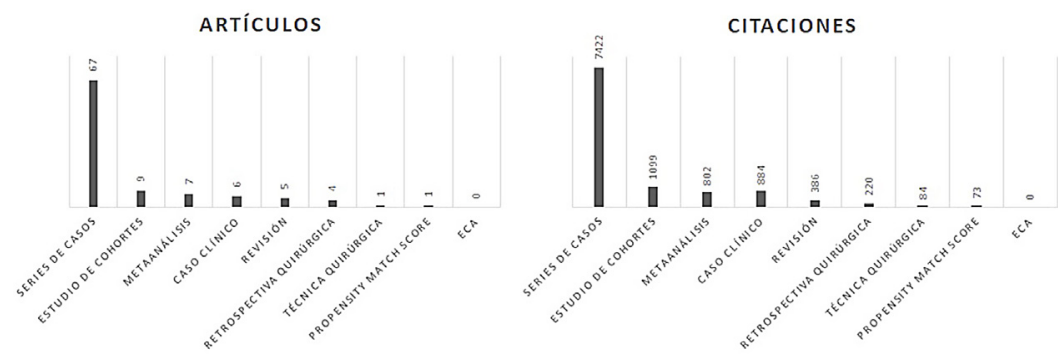

Figura 2 - Número de publicaciones y citaciones por tipo de papel en el top 100 de citas sobre páncreas y laparoscopia.

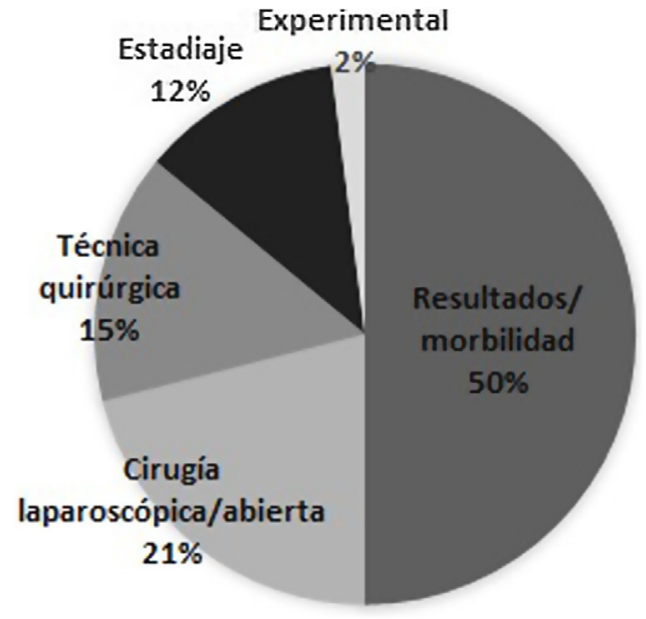

Figura 3 - Artículos según tema en el top 100 de citas sobre páncreas y laparoscopia.

2012, uno en 2013 y uno en 2015, 3 de ellos procedentes de China). De estos 7 metaanálisis, 6 comparan la PD abierta vs. laparoscópica y el otro compara PD y DP. No se incluyen ECA en el top 100 de citas (fig. 2).

El tema de resultados/morbilidad es el más estudiado en este top 100, con 5.218 citas y el $50 \%$ de las publicaciones, seguido por la comparación entre la cirugía laparoscópica y la abierta (21\% de las publicaciones, 2.278 citas) (fig. 3). Las lesiones benignas/malignas son el área de interés con el mayor número de publicaciones en el top 100 sobre páncreas y laparoscopia, mientras que no hay documentos sobre NMPI (tabla 3).

$\mathrm{Si}$ se excluyen 12 documentos que se refieren a la estadificación laparoscópica (fig. 3), el tipo de cirugía se recoge en la tabla 2. Treinta y tres artículos se refieren a la PD, con 3.488 citas, y seguido por 30 artículos sobre la cirugía pancreática en general; mientras que no hay referencias sobre la pancreatectomía total.

\section{Discusión}

El número de citas utilizadas como referencia en otros artículos se suele usar como medida del impacto de los trabajos científicos y como forma de validar las contribuciones de los autores ${ }^{1,4,6,9,10}$. Algunos autores afirman que, una vez que las ideas han sido generalmente aceptadas, los artículos más clásicos ${ }^{1-6}$ ya no se citan. Sin embargo, otros sostienen que el número de citas de un artículo refleja la duración de su vida académica, y especialmente el FI de la revista en la que se publicó ${ }^{4,9}$.

Este estudio bibliométrico sobre páncreas y laparoscopia está condicionado por el factor tiempo, ya que la laparoscopia ha llegado más tarde a la cirugía pancreática y este tema ha comenzado a estudiarse recientemente. Así pues, solo 19 artículos se publicaron antes del año 2000.

Desde el primer artículo incluido en este estudio bibliométrico publicado en 1978 por Cuschieri sobre la laparoscopia en el diagnóstico del cáncer de páncreas, el artículo tipo más citado es una serie de casos procedente de EE. UU. sobre el resultado/morbilidad de la $\mathrm{PD}$, que incluya lesiones benignas y malignas.

Surgical Endoscopy es la revista con más artículos incluidos en esta lista de los 100 más citados. Por lo tanto, los artículos sobre páncreas y laparoscopia tienden a publicarse en revistas más especializadas que en generalistas.

Tabla 3 - Número de artículos y citaciones según tipo de cirugía y área de interés en el top 100 de citas sobre páncreas y laparoscopia

\begin{tabular}{lll} 
Área de interés & Artículos & Citaciones \\
\hline Lesiones benignas/malignas & 44 & 5.129 \\
Tumor neuroendocrino & 15 & 1.358 \\
Adenocarcinoma & 13 & 1.475 \\
Enfermedad benigna & 10 & 1.498 \\
Neoplasias & 8 & 773 \\
Pancreatitis crónica & 4 & 348 \\
Pancreatitis aguda & 3 & 244 \\
Neoplasias quísticas & 1 & 145 \\
NMPI & 0 & 0 \\
& & \\
Tipo de cirugía & Artículos & Citaciones \\
\hline Pancreatectomía distal & 33 & 3.488 \\
Cirugía pancreática & 30 & 2.928 \\
Duodenopancreatectomía & 10 & 1.822 \\
Cirugía robótica & 7 & 717 \\
Cirugía paliativa & 4 & 312 \\
VARD & 3 & 244 \\
NR & 1 & 129 \\
Pancreatectomía total & 0 & 0 \\
\hline
\end{tabular}

NMPI: neoplasia mucosa papilar intraductal; NR: no referenciado; VARD: desbridamiento retroperitoneal videoasistido. 
En cuanto al tipo de artículo, hay que destacar la presencia de 7 metaanálisis en esta lista, sin ningún ECA incluido en el top 100 , lo que hace remarcable la relación dispar entre metaanálisis y ECA en este ámbito de trabajo. Revisados los metaanálisis incluidos en este ranking, la mayoría de ellos incluyen estudios comparativos no aleatorios, también incluidos en esta lista, 6 de los 7 comparan la PD abierta vs. laparoscópica y 3 proceden de China, donde existe un auge de este tipo de publicaciones. Recientemente, se han publicado algunos ECA relevantes en este campo, como el de Poves et al., del Hospital del Mar en Barcelona, en $2018^{11}$, quienes realizan una comparación entre la DP laparoscópica y abierta, con una estancia hospitalaria más corta y resultados postoperatorios más favorables con la laparoscopia, sin diferencias en ganglios linfáticos resecados o en márgenes de resección, con solo 15 citas hasta la fecha, y el de De Rooij et al. ${ }^{12}$, quienes publicaron el ensayo LEOPARD en 2019 e informaron de que la PD mínimamente invasiva reduce el tiempo de recuperación funcional en los tumores pancreáticos izquierdos, con menos retraso en el vaciamiento gástrico y una mejor calidad de vida (citado 19 veces). Además, van Hilst et al. publicaron en 2019 un «propensity score» paneuropeo que compara la PD mínimamente invasiva con la $\mathrm{PD}$ abierta y concluyeron que se necesitan ECA para confirmar la seguridad oncológica de la PD mínimamente invasiva ${ }^{13}$. En vista de la falta de estudios relevantes y científicamente sólidos, hay nuevos estudios dirigidos a llenar este vacío, como el ensayo LEOPARD 2 registrado en marzo de 2016 por el Grupo Holandés de Cáncer de Páncreas (citado 19 veces) ${ }^{14}$, y el ensayo COSMOS-DP en Japón registrado en julio de 2016 (citado una vez) ${ }^{15}$.

En cuanto al área de interés, no hay publicaciones sobre la pancreatectomía total o el NMPI. Por lo tanto, este estudio bibliométrico nos puede ayudar a identificar las áreas deficitarias que pueden ser objeto de nuevos estudios.

En nuestro estudio, EE. UU. es el primer país con 35 trabajos en el top 100 de citas sobre páncreas y laparoscopia. En comparación con otros estudios bibliométricos sobre cirugía no relacionada con el páncreas ${ }^{1,10}$, en nuestro estudio hay un menor predominio de EE. UU. frente al resto del mundo.

Nuestro artículo es un estudio bibliométrico realizado con medidas clásicas como el número de citaciones, medida que permite conocer cómo se obtiene la información científica, de dónde procede y cuál es su calidad. El principal problema es que los recuentos de citaciones tienen un período de retraso antes de que se pueda determinar el verdadero impacto de un artículo, ya que se tarda aproximadamente 2-3 años después de la publicación para que un artículo alcance el pico de citaciones $^{16,17}$, lo que retrasa la determinación del impacto de un artículo. Aunque las medidas tradicionales siguen siendo la norma para evaluar el efecto a largo plazo que ejerce una investigación, hoy en día, la revolución digital ha tenido un gran impacto en todos los campos profesionales y en la forma de medir sus repercusiones, una consecuencia natural de la digitalización que también afecta al campo de la difusión médica. Con ello, han surgido nuevas medidas alternativas, denominadas colectivamente «almetrics», como las descargas, los medios sociales como Facebook $₫$, Twitter $\AA$, LinkedIn $₫$, Pinterest $\AA$ o YouTube $\AA$, los recursos digitales como blogs o medios de noticias, las redes profesionales o los instrumentos de bibliografía, como medio no tradicional de evaluar la visibilidad de la publicación, un reflejo de la popularidad y el debate social a corto plazo.

En este debate entre medidas tradicionales y nuevas, hay que tener en cuenta el destino de las publicaciones, ya que el acceso a las plataformas online está abierto al público general. Así pues, un artículo puede suscitar gran interés en los lectores, pero poco impacto académico porque los lectores han sido en su mayoría público en general y no profesionales de la salud. Por lo tanto, debemos considerar que la disponibilidad del documento influye en su impacto; los artículos publicados en revistas de libre acceso online están ampliamente disponibles para el consumo del público y están aumentando su visibilidad a través de diversos canales de comunicación ${ }^{18}$, mientras que los publicados en revistas que requieren pago están orientados hacia el mundo académico. Por tanto, en la era tecnológica en la que estamos, además del número de citas, hay que considerar otros factores digitales para evaluar el impacto de un investigador o un artículo.

La cirugía laparoscópica ha llegado tarde al páncreas, probablemente debido a la morbimortalidad asociada a la cirugía pancreática y a la necesidad de una alta especialización en este campo. Esto significa que la información de la que se dispone es reciente y escasa. Se necesitan más estudios y de mayor calidad en este campo.

\section{Conflicto de intereses}

Los autores declaran no tener ningún conflicto de intereses.

\section{Anexo. Material adicional}

Se puede consultar material adicional a este artículo en su versión electrónica disponible en XXXXXXXXXX.

\section{B I B L I O G R A F Í A}

1. Paladugu R, Schein M, Gardezi S, Wise L. One hundred citation classics in general surgical journals. World J Surg. 2002;26:1099-105.

2. Azer SA, Azer S. Bibliometric analysis of the top cited gastroenterology and hepatology articles. BMJ Open. 2016;6:e009889.

3. Tang X, Gong W, Yuan F, Li R, Han X, Huang S, et al. Topcited articles in digestive disease from 1950 to 2013. J Gastroenterol Hepatol. 2016;31:107-11.

4. Ellul T, Bullock N, Abdelrahman T, Powell A, Witherspoon J, Lewis WG. The 100 most cited manuscripts in emergency abdominal surgery: A bibliometric analysis. Int J Surg. 2017;37:29-35.

5. Ahmad S, Ahmad S, Kohl S, Ahmad S, Ahmed AR. The hundred most cited articles in bariatric surgery. Obes Surg. 2015;25:900-9.

6. O'Sullivan KE, Hurley JP. The 100 most cited publications in transplantation. Ann Transplant. 2014;19:436-43.

7. Uhtman OA, Okwundu CI, Wiysonge CS, Young T, Clarke A. Citation classics in systematic review and meta-analyses: who wrote the top 100 most cited articles? PLoS One. 2013;8:e78517.

8. Cucchetti A, Mazzotti F, Pellegrini S, Cescon M, Maroni L, Ercolani G, et al. The use of the Hirsch index in 
benchmarking hepatic surgery research. Am J Surg. 2013;206:560-6.

9. Long X, Huang JZ, Ho YSH. A historical review of classical articles in surgery field. Am J Surg. 2014;208:841-9.

10. Manuel Vázquez A, Latorre Fragua R, López Marcano A, Ramiro Pérez C, Arteaga Peralta V, de la Plaza-Llamas R, et al. The top 100: A review of the most cited articles in Surgery. Cir Esp. 2019;97:150-5.

11. Poves I, Burdío F, Morató O, Iglesias M, Radosevic A, Ilzarbe $\mathrm{L}$, et al. Comparison of perioperative outcomes between laparoscopic and open approach for pancreatoduodenectomy: The PADULAP randomized controlled trial. Ann Surg. 2018;268:731-9.

12. De Rooij T, van Hilst J, van Santvoort H, Boerma D, van den Boezem P, Daams F, et al., Dutch Pancreatic Cancer Group. Minimally invasive versus open distal pancreatectomy (LEOPARD): a multicenter patient-blinded randomized controlled trial. Ann Surg. 2019;269:2-9.

13. Van Hilst J, de Rooij T, Klompmaker S, Rawashdeh M, Aleotti F, Al-Sarireh B, et al., European Consortium on Minimally Invasive Pancreatic Surgery (E-MIPS). Minimally invasive versus open distal pancreatectomy for ductal adenocarcinoma (DIPLOMA): a pan-European propensity score matched study. Ann Surg. 2019;269:10-7.

14. De Rooij T, van Hilst J, Bosscha K, Dijkgraaf MG, Gerhards MF, Groot Koerkamp B, et al., Dutch Pancreatic Cancer Group. Minimally invasive versus open pancreatoduodenectomy (LEOPARD-2): study protocol for a randomized controlled trial. Trials. 2018;19:1.

15. Yamada S, Fujii T, Kawai M, Shimokawa T, Nakamura M, Murakami $Y$, et al. Splenic vein resection together with the pancreatic parenchyma versus separated resection after isolation of the parenchyma during distal pancreatectomy (COSMOS-DP trial): study protocol for a randomised controlled trial. Trials. 2018;19:369.

16. Trueger NS, Thoma B, Hsu CH, Sullivan D, Peters L, Lin M. The altmetric score: a new measure for article level dissemination and impact. Ann Emerg Med. 2015;66:549-53.

17. Wilsdon J, Allen L, Belfiore E, Campbell P, Curry S, Hill S, et al. The Metric Tide: report of the Independent Review of the Role of Metrics in Research Assessment and Management. Bristol: Higher Education Funding Council for England. 2015

18. Van Noorden R. Open access: The true cost of science publishing. Nature. 2013;495:426-9. 